\title{
Development of an atomic-force-microscope-based hanging-fiber rheometer for interfacial microrheology
}

\author{
Xiaomin Xiong, ${ }^{1,2}$ Shuo Guo, ${ }^{1}$ Zuli Xu, ${ }^{1}$ Ping Sheng, ${ }^{1}$ and Penger Tong ${ }^{1, *}$ \\ ${ }^{1}$ Department of Physics, Hong Kong University of Science and Technology, Clear Water Bay, Kowloon, Hong Kong \\ ${ }^{2}$ Department of Physics and State Key Laboratory of Optoelectronic Materials and Technologies, Sun Yat-sen University, \\ Guangzhou, China
}

(Received 2 May 2009; revised manuscript received 6 August 2009; published 30 December 2009)

\begin{abstract}
A new interfacial microrheology technique using atomic force microscope (AFM) as a force sensor is developed. The probe used for microrheology contains a long vertical glass fiber with one end glued onto a rectangular shaped cantilever beam and the other end immersed through a water-air interface. The motion of the modified cantilever can be accurately described by the Langevin equation for a damped harmonic oscillator, from which we obtain the friction coefficient $\xi$ of the glass fiber in contact with the water. It is found that $\xi$ contains two contributions. One is generated by the bulk fluid, which increases with the immersion length of the glass fiber. The other contribution comes from the contact line between the water-air interface and the glass fiber, which is obtained by an extrapolation of the measured $\xi$ at the limit of zero immersion length. The experiment thus demonstrates an application of AFM in the studies of interfacial microrheology and contact line dynamics.
\end{abstract}

DOI: 10.1103/PhysRevE.80.061604

\section{INTRODUCTION}

Interfaces play a vital role in technological applications in such diverse areas as catalysis, lubrication, printing, material synthesis, microfluidics, and nanotechnology [1]. Moreover, the study of interfacial dynamics and interactions is inherently connected to cell biology. Many biochemical reactions and intracellular signaling that sustain life occur at interfaces. For example, diffusive transport of lipids and membrane-bound proteins plays an important role in many aspects of cell biology $[2,3]$. For these reasons, the past decade has seen a growing interest in the use of the unique environment of interfaces to explore fascinating science and new applications.

Atomic force microscope (AFM) and related scanning probe microscope have proved to be a powerful tool for the experimental investigation of interfaces. They have been used recently to measure the lubrication forces near a solid wall [4-6] and the capillary forces at a liquid-air interface [7]. The AFM operates by measuring the deflection signal of a cantilever resulting from interactions between a sharp tip at the further end of the cantilever beam and the sample surface. In the tapping mode, the AFM cantilever oscillates near its own resonant frequency and the change of the cantilever's oscillation amplitude due to the interaction force between the tip and sample surface is used as the feedback. The tapping mode is particularly useful for investigations of sample surfaces in air, in which the viscous damping is small and the cantilever's resonant peak is sharp with a large value of quality factor $Q$ [8].

In recent years, there has been a growing interest in the AFM application in liquid environments for the study of biomolecular structures [9] and dynamics near solid-liquid and liquid-liquid interfaces [10-12]. When the AFM cantilever is immersed in a liquid, its motion (e.g., oscillations in the

\footnotetext{
*penger@ust.hk
}

PACS number(s): 68.05.-n, 07.79.-v, 83.10.Mj, 83.85.Vb

tapping mode) is damped by the fluid viscosity. The mechanical excitation of the cantilever by a piezo-electric shaker also generates different flow modes near the surface, which may in turn alter the resonance spectrum of the cantilever. A number of theoretical and numerical studies have been carried out aimed at understanding the mechanical response of small cantilevers of different geometry immersed in a viscous fluid $[8,12-17]$. Experimental efforts were also made to increase the $Q$ value by using active feedback circuits [18]. Such an electronic feedback, however, still requires a detectable resonant signal to begin with. An intriguing proposal arriving from these studies is to use AFM as a passive rheometer for the study of microrheology of complex fluids $[8,12,15,17,19]$. The proposal was based on the application of a known oscillatory strain on the fluid surrounding the cantilever. The dissipative or viscoelastic response of the fluid is then obtained from the measured power spectrum of the cantilever in response to the strain imparted by thermal fluctuations. This technique was named as Brownian fluctuation spectroscopy by Ma et al. [17].

Contrary to the large number of theoretical and numerical studies, however, the experimental implementation of the technique turns out to be quite difficult. First, because the cantilever is fully immersed in the liquid, its motion is often overdamped. This is particularly true when the cantilever is placed near a solid surface [10]. As a result, the power spectrum of cantilever deflections flattens out, making the measurement of the cantilever's resonant peak inaccurate. Second, like any standard rheometer, a quantitative analysis of the rheological measurements requires a known strain field to be generated by a simple geometry. The use of AFM cantilevers with different shapes as a probe introduces further complications and uncertainties for the quantitative description of the rheological properties of complex fluids [19].

In this paper we report the development of an AFM-based technique to overcome these experimental difficulties. In the technique, we design an AFM probe with simpler geometry 
for the study of interfacial microrheology. The modified AFM probe contains a long hanging glass fiber with one end glued on the frontend of the cantilever beam. The hanging glass fiber is partially immersed through a liquid-air interface, leaving the cantilever beam entirely in air. Such a design allows us to precisely measure the drag force on the thin glass fiber without introducing an overdamping to the entire system. It also simplifies the theoretical analysis of the rheological measurements at the liquid-air interface and thus provides a useful tool for the study of viscous dissipations near the contact line between the interface and the glass fiber.

The paper is organized as follows. First, we describe the basic working principle of the technique. Second, we report the experimental results, which verify the method and illustrate its applications. A primary objective of the paper is to delineate the experimental conditions for the precise measurement of the viscous drag on the thin glass fiber. The experiment demonstrates that the AFM-based passive rheometer has the sensitivity and accuracy for the measurement of the extra drag produced by the contact line between the glass fiber and the liquid-air interface. We expect this technique to be useful in the future study of interfacial dynamics of complex fluids.

\section{THEORETICAL BACKGROUND}

In this section, we discuss the working principle of the cantilever probe with a hanging glass fiber partially immersed through a liquid-air interface. Figure 1 shows a schematic drawing of the modified cantilever and the AFM setup for the measurement of the interactions between the hanging glass fiber and the liquid-air interface. When a vertical glass fiber is partially immersed through a liquid-air interface, there exists a circular contact line on the fiber surface (dashed circle shown in Fig. 1). The force acting on the glass fiber causes the flexible cantilever to bend and its vertical displacement is monitored by a reflecting (red) laser beam together with an intercepting position-sensitive photodetector.

The basic theory for the motion of the AFM cantilever in a liquid environment has been discussed previously in the literature $[8,12,15,17]$. For a thin glass fiber, its vertical motion can be described by the Langevin equation $[12,17]$,

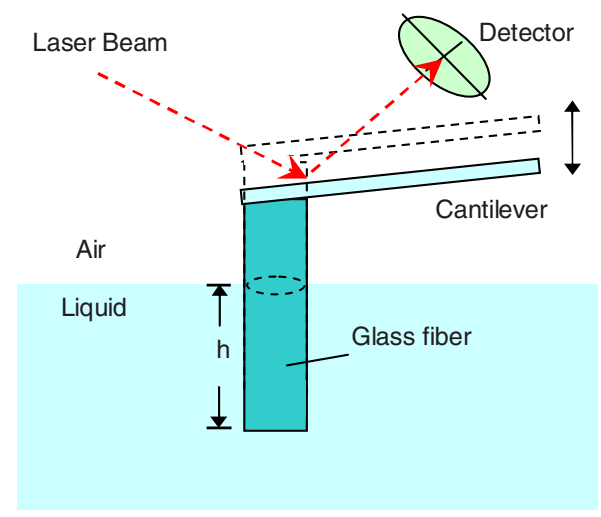

FIG. 1. (Color online) A schematic drawing (not to the scale) of the modified cantilever with a vertical hanging glass fiber partially immersed through a liquid-air interface and the AFM setup for the measurement of hydrodynamic interactions between the hanging glass fiber and the liquid-air interface.

$$
m \frac{\partial^{2} z}{\partial t^{2}}+\xi \frac{\partial z}{\partial t}+k z=f_{e x t}(z)+f_{B}(t),
$$

where $z(t)$ is the vertical displacement of the glass fiber at time $t$ ( $\equiv$ vertical deflection of the cantilever), $\xi \partial z / \partial t$ is the drag force on the glass fiber with $\xi$ being the friction coefficient, $k z(t)$ is the elastic force due to the bending of the cantilever with a spring constant $k$, and $f_{\text {ext }}(z)$ accounts for other external forces acting on the modified cantilever. Hereafter, we assume $f_{\text {ext }}(z)$ to be zero for simplicity. The last force $f_{B}(t)$ is the random Brownian force due to thermal fluctuations of the surrounding fluid. While the mean value of $f_{B}(t)$ is zero, its autocorrelation function, $C(\tau)$ $=\left\langle f_{B}(t+\tau) f_{B}(t)\right\rangle$, is nonzero and has a form [20]

$$
C(\tau)=2 k_{B} T \xi \delta(\tau),
$$

where $k_{B} T$ is the thermal energy of the system and $\delta(t)$ is the $\delta$ function. While deviations from Eq. (2) may arise in complicated situations, such as thermal motions of a microscale oscillator with complex geometry or a microcantilever near a solid wall $[14,15]$, we consider here a simple case of a long glass fiber with only one resonant mode excited. In this case, Eq. (2) is chosen as an approximate form for simplicity.

The measurable quantity in the experiment is the power spectrum, $|z(\omega)|^{2}$, of vertical deflections of the cantilever (or equivalently $|z(f)|^{2}$ ), which can be solved analytically from Eq. (1) $[17,21]$,

$$
|z(\omega)|^{2}=\frac{2 k_{B} T \xi / m^{2}}{\left(\omega^{2}-\omega_{0}^{2}\right)^{2}+(\omega \xi / m)^{2}}=\frac{2 k_{B} T \xi / m^{2}}{\left\{\omega^{2}-\left[\omega_{0}^{2}-(1 / 2)(\xi / m)^{2}\right]\right\}^{2}+(\xi / m)^{2}\left[\omega_{0}^{2}-(1 / 4)(\xi / m)^{2}\right]},
$$

where $\omega=2 \pi f$ is the angular frequency and $\omega_{0}=(k / m)^{1 / 2}$ is the resonant frequency of the system. In obtaining Eq. (3), one has made a number of simplifications. In Sec. IV, we will present the experimental results and demonstrate that the motion of the hanging glass fiber at the interface can indeed be described by Eq. (3).

An important result is obtained by integrating $|z(\omega)|^{2}$ in Eq. (3) over the entire frequency domain, 


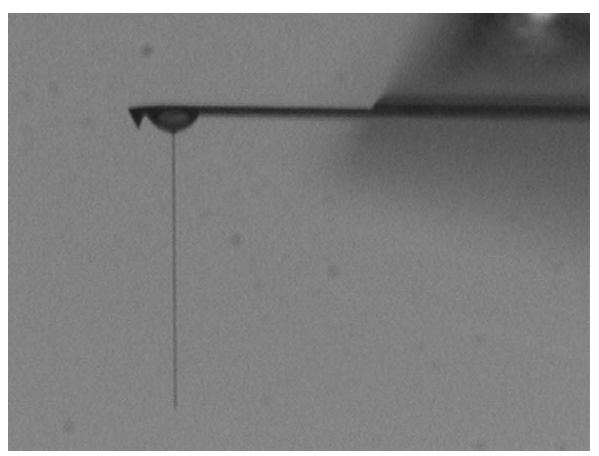

FIG. 2. A modified AFM cantilever made in our laboratory with a hanging glass fiber with diameter of $d=2 \mu \mathrm{m}$ and length of $280 \mu \mathrm{m}$. The top of the glass fiber is glued onto a rectangular shaped cantilever beam.

$$
\begin{aligned}
\left\langle z^{2}(t)\right\rangle_{t}=\int_{-\infty}^{\infty}|z(f)|^{2} d f & =2 \int_{0}^{\infty} \frac{2 k_{B} T \xi / m^{2}}{\left(\omega^{2}-\omega_{0}^{2}\right)^{2}+(\omega \xi / m)^{2}} \frac{d \omega}{2 \pi} \\
& =\frac{k_{B} T}{k},
\end{aligned}
$$

where $\left\langle z^{2}(t)\right\rangle_{t}$ is the mean square value of the vertical deflections $z(t)$. Equation (4) states that the elastic energy per mode, $(1 / 2) k\left\langle z^{2}(t)\right\rangle_{t}$, stored in the cantilever is provided by the thermal energy $(1 / 2) k_{B} T$ and is independent of viscous damping.

\section{EXPERIMENT}

Figure 2 shows a modified cantilever made for the experiment with a vertical hanging glass fiber of $2 \mu \mathrm{m}$ in diameter and $280 \mu \mathrm{m}$ in length. The thin glass fiber is made from a glass rod of $2 \mathrm{~mm}$ in diameter and $100 \mathrm{~mm}$ in length. A pippette puller (Model P-97, Sutter Instrument Co.) is used to pull the glass rod into a thin fiber. The glass rod is cleaned with a $\mathrm{H}_{2} \mathrm{SO}_{4} / \mathrm{H}_{2} \mathrm{O}_{2}$ mixture solution prior to the pulling. The glass fiber is then glued onto the further end of a rectangular shaped cantilever beam with width of $50 \mu \mathrm{m}$, thickness of $2 \mu \mathrm{m}$, and length in the range of 130-200 $\mu \mathrm{m}$. Using the perpendicular lines of a ruler in the eye piece, we align the glass fiber to be perpendicular to the cantilever beam under a microscope. It is found that such a visual alignment is adequate to ensure that there is only one resonant mode excited in the wide frequency range as shown in Fig. 3. In fact, we find that for the glass fiber used in the experiment, there will be no other unwanted resonant mode excited if the fiber's angular deviation with respect to the vertical position is smaller than $15^{\circ}$. Commercial silicon microcantilevers (MikroMasch) with spring constant $k$ in the range of $2-16 \mathrm{~N} / \mathrm{m}$ are used in the experiment. While a shorter beam length increases the detection sensitivity of cantilever deflection [22], a spare space is needed to glue the glass fiber onto the cantilever beam. The glue is cured via the radiation of ultraviolet light. Finally, the glass fiber is cut to a desired length, typically $\sim 300 \mu \mathrm{m}$, using a pair of homemade sharp tweezers. The entire assembly process is carried out with the help of a motorized micromanipulator system

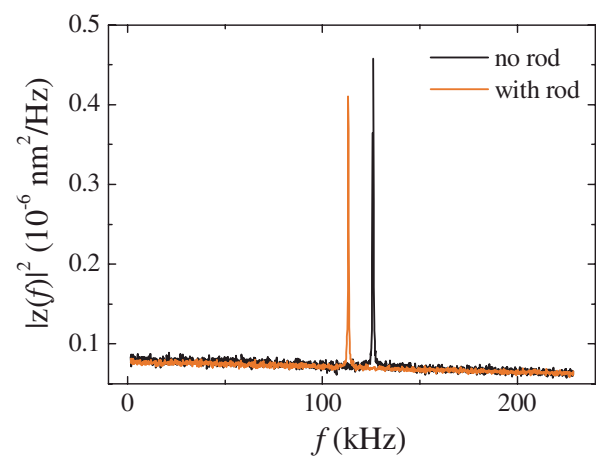

FIG. 3. (Color online) Comparison of the measured power spectra $|z(f)|^{2}$ as a function of $f$ for a bare cantilever [right (black) peak] and the modified cantilever with a hanging glass fiber of diameter $d=2 \mu \mathrm{m}$ [left (red) peak]. The measurements are made in air.

together with a high-magnification stereomicroscope (Leica MZ16).

The water-air interface is prepared using a sample cell made from a stainless steel disk, which is hydrophilic and has a central well with diameter of $10 \mathrm{~mm}$ and depth of 5 $\mathrm{mm}$. The top of the well has a sharp circular edge, which is used to pin the water-air interface in order to reduce unwanted surface flow. The diameter of the well is chosen to be not too small so that the water-air interface can be kept flat visually. It should also be not too large in order to reduce unwanted surface flow. Similarly, we choose the water layer to be thick enough so that the motion of the glass fiber will not be influenced by the cell boundaries. The entire stainless steel disk is mounted inside a closed AFM fluid cell purchased from Asylum Research Inc. Using a flexible rubber diaphragm, the fluid cell seals the AFM cantilever and the sample cell in a closed chamber.

Prior to each measurement, the sample cell is thoroughly cleaned following the same procedures as described in Ref. [23]. De-ionized water (purified with a Barnstead three column $e$-pure system to a resistivity of $18 \mathrm{M} \Omega . \mathrm{cm}$ ) is filled to the sample cell by a syringe and then the entire fluid cell is sealed to minimize the evaporation of the fluid. A stepper motor is used to move the bottom part of the fluid cell upward with an accuracy of $0.1 \mu \mathrm{m}$. When the tip of the glass fiber touches the water-air interface, a large change in the cantilever deflection is detected by an AFM sensor, making the determination of the contact point between the glass fiber tip and the water-air interface to be accurate to within $1 \mu \mathrm{m}$. In the experiment, the modified cantilever (including the glass fiber) remains stationary and the stepper motor is used to move the interface vertically toward the glass fiber in steps of $1 \mu \mathrm{m}$. The immersion length $h$ of the glass fiber into the water-air interface (see Fig. 1) can be varied in the range $1-300 \mu \mathrm{m}$.

Measurements of the power spectrum $|z(f)|^{2}$ are conducted using an AFM (MFP-3D, Asylum Research Inc.) operated under the thermal power spectral density (PSD) mode. Under this mode, the voltage signal from the position sensitive-detector (see Fig. 1) is digitized at a sampling frequency $5 \mathrm{MHz}$. Typically, $|z(f)|^{2}$ is taken with a frequency resolution of $152 \mathrm{~Hz}$ and the average time for each $|z(f)|^{2}$ at a fixed value of $h$ is set for $2-5 \mathrm{~min}$. To determine the abso- 


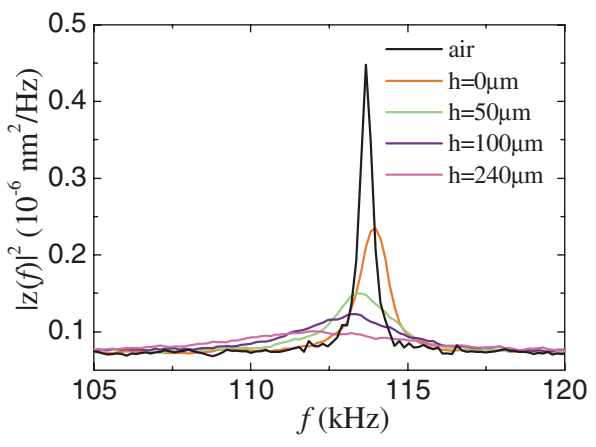

FIG. 4. (Color online) Evolution of the measured $|z(f)|^{2}$ when the glass fiber is pushed through a water-air interface at different immersion lengths (from right to left): in air (black curve), $h$ $=0 \mu \mathrm{m}$ (just in touch with the interface, red curve), $h=45 \mu \mathrm{m}$ (green curve), $h=95 \mu \mathrm{m}$ (dark blue curve), and $h=235 \mu \mathrm{m}$ (light magenta curve).

lute value of $|z(f)|^{2}$, the output voltage signal from the position-sensitive detector is calibrated against known values of the cantilever deflection. This is done for all the bare cantilevers without a hanging glass fiber using the procedure provided by the AFM manufacturer. For the modified cantilevers with a hanging glass fiber, we assume their calibration constant remains the same as that without a hanging glass fiber. Using this procedure, we obtain the material parameters of the modified cantilever, such as its spring constant, by calibrating the fiber-attached cantilever in air. It is found that the experimental uncertainties of the measured $|z(f)|^{2}$ can be kept at the level of 5\%-10\%.

\section{EXPERIMENTAL RESULTS}

Figure 3 compares the measured power spectra $|z(f)|^{2}$ as a function of $f$ for a bare cantilever [right (black) peak] and the modified cantilever with a hanging glass fiber of diameter $2 \mu \mathrm{m}$ [left (red) peak]. Over a wide frequency range up to $200 \mathrm{kHz}$, the measured $|z(f)|^{2}$ shows only one resonant peak, indicating that the motion of the modified cantilever can indeed be described by a simple harmonic oscillator with an effective mass $m$, friction coefficient $\xi$ and spring constant $k$, as shown in Eq. (1). Because of the increase in mass, the resonant frequency of the modified cantilever is reduced. There is a smooth noise background, which decreases slightly with increasing $f$. This noise background is produced primarily by the electronic noise of the system and thermal drifts of the AFM laser source.

Figure 4 shows how the measured $|z(f)|^{2}$ changes when the hanging-fiber is pushed through the water-air interface at different immersion lengths $h$. When the glass fiber touches the water-air interface, its resonant frequency increases slightly from 113.67 to $113.98 \mathrm{kHz}$. This small change $(\sim 0.27 \%)$ in the resonant frequency is caused by the increase of the effective spring constant $k$ of the entire system, which consists of two terms, $k=k_{c}+k_{s}$, with $k_{c}$ being the spring constant of the cantilever and $k_{s}$ being the surface tension induced spring constant due to the pinning of the interface to the fiber surface. We find that a value of $k_{c}$

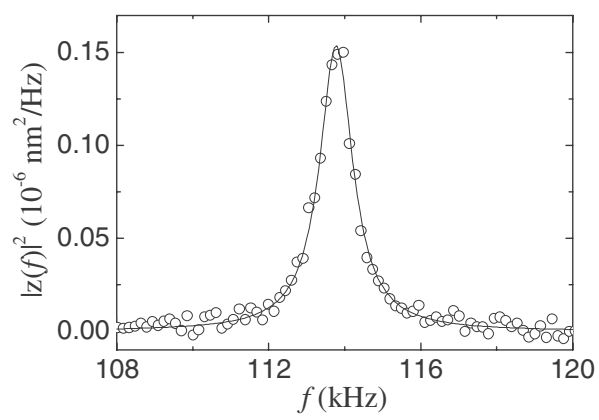

FIG. 5. A magnified plot of the measured $|z(f)|^{2}$ as a function of $f$ at $h \simeq 10 \mu \mathrm{m}$. The solid line shows a fit to Eq. (3) with $m$ $=3.16 \times 10^{-8} \mathrm{~g}, k=16.1 \mathrm{~N} / \mathrm{m}$, and $\xi=0.211 \times 10^{-6} \mathrm{~N} \mathrm{~s} / \mathrm{m}$.

$\simeq \gamma / 2$ can account for the $0.27 \%$ increase in the resonant frequency [24]. Here $\gamma \simeq 72 \mathrm{mN} / \mathrm{m}$ is the surface tension of the water-air interface. Once in touch with the water-air interface, the frequency peak changes continuously with increasing damping (i.e., with increasing values of $h$ ); the frequency peak broadens while the peak height decreases and peak position shifts to lower frequencies. This behavior agrees with the prediction shown in the second equality of Eq. (3). Figure 4 thus demonstrates that the measured $|z(f)|^{2}$ is very sensitive to the viscous damping and one can use this sensitivity to study the microhydrodynamics near liquid-air interfaces.

Figure 5 shows a magnified plot of the measured $|z(f)|^{2}$ as a function of $f$ at the immersion length $h \simeq 10 \mu \mathrm{m}$. The solid line is a fit to Eq. (3), which describes the data well with three fitting parameters: the mass $m=3.16 \times 10^{-8} \mathrm{~g}$, spring constant $k=16.1 \mathrm{~N} / \mathrm{m}$, and friction coefficient $\xi=0.211$ $\times 10^{-6} \mathrm{~N} \mathrm{~s} / \mathrm{m}$. There are two additional fitting parameters used to describe the slow linear decay of the noise background with increasing $f$. This noise background has been subtracted out from the data shown in Fig. 5. With the measured $|z(f)|^{2}$, we conduct the numerical integration, $\left\langle z^{2}(t)\right\rangle_{t}$ $=2 \int_{0}^{\infty}|z(f)|^{2} d f$, over the entire frequency range and check the relationship shown in Eq. (4) [25]. It is found that Eq. (4) indeed holds for the measured $|z(f)|^{2}$ at the water-air interface with different values of $h$.

Figure 6 shows the fitted values of the spring constant $k$ for different immersion lengths $h$. The values of $k$ are obtained in two ways; one is obtained directly from the fitting (circles) and the other is obtained from the integration method using the equation $k=k_{B} T /\left\langle z(t)^{2}\right\rangle_{t}$ (triangles). For comparison, we include the value of $k \simeq 14.0 \mathrm{~N} / \mathrm{m}$ obtained in air in the plot and place it at $h=-10 \mu \mathrm{m}$. The values of $k$ obtained by the two methods agree well with each other. They remain approximately constant for different values of $h$. The dashed line indicates the mean value $\bar{k}=14.64 \mathrm{~N} / \mathrm{m}$, which is obtained by averaging over all the data points. The standard deviation of the measured $k$ is $\sigma=0.51 \mathrm{~N} / \mathrm{m}$, which is $3.5 \%$ of the mean value $\bar{k}$. We believe that some of the scatterers of the data shown in Fig. 6 are caused by the random pinning of the interface to the surface of the glass fiber [26-28]. As mentioned above, the effective spring constant $k$ of the system consists of two contributions, $k=k_{c}$ $+k_{s}$. Because the spring constant $k_{c}$ of the cantilever used in 


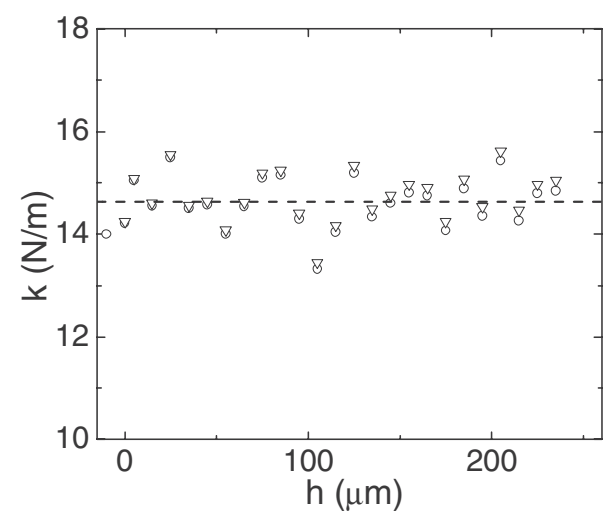

FIG. 6. Fitted values of the spring constant $k$ for different immersion lengths $h$. The values of $k$ are obtained in two ways; one is obtained directly from the fitting (circles) and the other is obtained from the integration method using the equation $k=k_{B} T /\left\langle z(t)^{2}\right\rangle_{t}$ (triangles). The dashed line indicates the mean value $\bar{k}=14.64 \mathrm{~N} / \mathrm{m}$.

the experiment is much larger than the surface tension induced spring constant $k_{s}$, the value of $k$ remains approximately the same as $k_{c}$, which does not change with $h$. Note that the rms value of $z(t)\left[\equiv\left(k_{B} T / \bar{k}\right)^{1 / 2}\right]$ corresponding to $\bar{k}$ is only $1.68 \times 10^{-2} \mathrm{~nm}$, which reveals the tremendous sensitivity of the technique.

Figure 7 shows the fitted values of the friction coefficient $\xi$ as a function of the immersion length $h$. Three sets of data obtained using three different modified cantilevers with spring constant $k \simeq 9 \mathrm{~N} / \mathrm{m}$ (triangles), $14 \mathrm{~N} / \mathrm{m}$ (circles), and $16 \mathrm{~N} / \mathrm{m}$ (diamonds), respectively, are shown here. The glass fibers used for the three modified cantilevers have approximately the same diameter $1.5 \pm 0.2 \mu \mathrm{m}$ and length $280 \pm 10 \mu \mathrm{m}$. The measured values of $\xi$ using the three different probes overlap well. The errors bars in the plot indicate the typical fitting uncertainties of each data point. With increasing viscous damping (i.e., with increasing values of

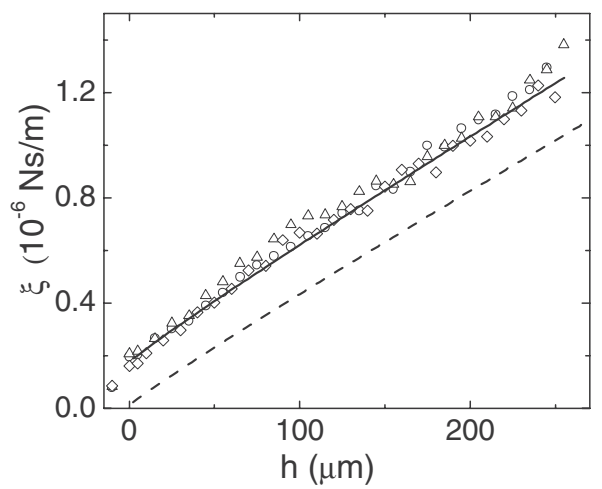

FIG. 7. Fitted values of the friction coefficient $\xi$ as a function of the immersion length $h$. The measurements are made using three different modified cantilevers with spring constant $k \simeq 9 \mathrm{~N} / \mathrm{m}$ (open triangles), $14 \mathrm{~N} / \mathrm{m}$ (open circles), and $16 \mathrm{~N} / \mathrm{m}$ (open diamonds). For comparison, the fitted values of $\xi$ in air are also included at $h=-10 \mu \mathrm{m}$. The solid line is a least-square fit of Eq. (9) to the diamonds with $\alpha=31.2 \pm 4$ and $\eta=1.08 \pm 0.08 \mathrm{cP}$. The dashed line is a plot of Eq. (5) using the calculated $\xi_{\|}^{0}$ for a long cylinder as shown in Fig. 8 and $\eta=1.0 \mathrm{cP}$. $h$ ), the frequency peak broadens while the peak height decreases, causing the uncertainty of the fitted values of $\xi$ to increase with $h$. The fitted values of $\xi$ at smaller values of $h$, on the other hand, are more sensitive to the minute amount of impurities at the interface. Overall, we find that the experimental uncertainties of the measured $\xi$ are at the $\sim 10 \%$ level.

Figure 7 reveals two interesting features of interfacial hydrodynamics. First, the measured $\xi$ at the $h \simeq 0$ limit has a finite value $\left[\xi(0) \simeq 0.19 \times 10^{-6} \mathrm{~N} \mathrm{~s} / \mathrm{m}\right]$, which is 2.3 times larger than that measured in air $\left(\xi \simeq 8.26 \times 10^{-8} \mathrm{~N} \mathrm{~s} / \mathrm{m}\right)$. For comparison, we include the fitted values of $\xi$ in air in the plot and place them at $h=-10 \mu \mathrm{m}$. Second, the measured $\xi(h)$ shows an approximate linear dependence on the immersion length $h$ for large values of $h$. In the following, we will show that (i) a significant contribution to the measured $\xi(0)$ comes from the viscous dissipation of the (fluctuating) contact line between the water-air interface and the glass fiber. (ii) The deviations from the linear dependence on $h$ at small values of $h$ result from the dependence of the measured $\xi$ on the diameter of the glass fiber. (iii) The slope of the measured $\xi(h)$ at large values of $h$ is determined by a combined effect of zerofrequency friction and inertia of the oscillating fiber.

While at the moment we are not aware of any theoretical calculation for the viscous drag of a long cylinder across a liquid-air interface, the friction coefficient $\xi$ of a slender body in an infinite oscillating flow without boundaries is known [29-32]. For a long oscillating cylinder of length $h$ and diameter $d$ fully immersed in an infinite liquid of viscosity $\eta$, its friction coefficient $\xi_{\|}$parallel to the long axis of the cylinder is given by $[17,31]$

$$
\xi_{\|}=\xi_{\|}^{0}+\pi(d / 2) h(2 \eta \rho \omega)^{1 / 2},
$$

where $\xi_{\|}^{0}$ represents the zero-frequency friction coefficient and $\rho$ is the density of the fluid [33]. For simple geometry, such as a prolate spheroid, the analytic form of $\xi_{\|}^{0}$ is known as [29]

$$
\xi_{\|}^{0}=8 \pi \eta h \frac{e^{3}}{\left(1+e^{2}\right) \ln [(1+e) /(1-e)]-2 e},
$$

where $e=\left[1-(d / h)^{2}\right]^{1 / 2}$ is the eccentricity of the spheroid. When $d / h=\left(1-e^{2}\right)^{1 / 2} \ll 1, \xi_{\|}^{0}$ takes the limiting form [29]

$$
\xi_{\|}^{0}=\frac{2 \pi \eta h}{\ln (h / d)+\gamma},
$$

with $\gamma \simeq 0.193$. Except the logarithmic correction factor, $\xi_{\|}^{0}$ (and thus $\xi_{\|}$) is a linear function of $h$ for large values of $h$. Equations (5) and (7) thus explain the linear behavior of the measured $\xi$ at large values of $h$.

For a long glass fiber used in the experiment, the analytic form of $\xi_{\|}^{0}$ is not available at the moment. To investigate the geometry effect on the friction coefficient, we calculate $\xi_{\|}^{0}$ for a long solid cylinder of length $h$ and diameter $d$ $=1.5 \mu \mathrm{m}$ fully immersed in a large volume of liquid. The numerical calculation is carried out by solving the steadystate incompressible Navier-Stokes equation for a creeping flow of velocity $v_{0} \simeq 10 \mu \mathrm{m} / \mathrm{s}$ passing over a stationary cylinder, whose long axis is parallel to the flow direction. The 


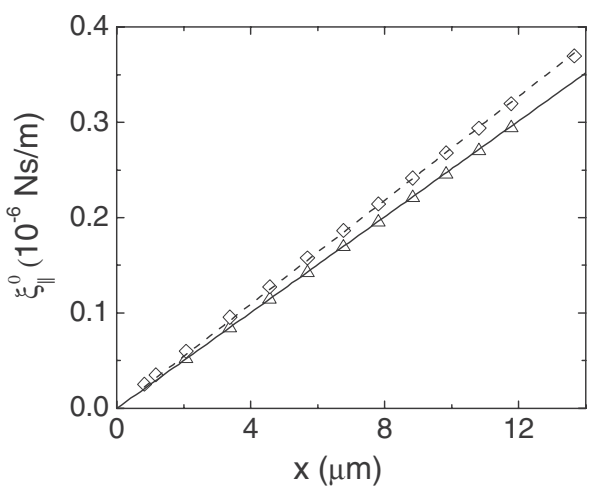

FIG. 8. Calculated friction coefficient $\xi_{\|}^{0}$ as a function of $x$ for a prolate spheroid (triangles) and a long solid cylinder (diamonds). The diameter of the spheroid and the cylinder used in the numerical calculation is $1.5 \mu \mathrm{m}$. The solid line is a plot of Eq. (6) with the slope $8 \pi \eta \simeq 25.12 \mathrm{cP}$ (for water). The dashed line is a linear fit, $\xi_{\|}=c 8 \pi \eta x$, to the diamonds with $c \simeq 1.09$.

numerical model is implemented using the COMSOL multiphysics software package. Following the symmetry of the solid cylinder, we create a flow domain of the same cylindrical shape around the cylinder with the diameter and length more than $10^{2}$ times larger than that of the cylinder. The solid cylinder is placed at the center of the flow domain and noslip boundary conditions (i.e., zero velocity) are applied on the surface of the cylinder. The outer surface of the flow domain is set with the same $v_{0}$ all around. We have verified that the calculated $\xi_{\|}^{0}$ changes less than $0.2 \%$, when the size of the flow domain is further increased by a factor of 10 .

Figure 8 shows the numerically calculated $\xi_{\|}^{0}$ as a function of a new variable,

$$
x=h \frac{e^{3}}{\left(1+e^{2}\right) \ln [(1+e) /(1-e)]-2 e},
$$

for a prolate spheroid (triangles) and a long cylindrical rod (diamonds). This combined length variable removes the apparent dependence on the diameter (or aspect ratio) of the glass fiber. The numerical results for the spheroid agree well with the analytic solution $\xi_{\|}^{0}=8 \pi \eta x$ (solid line) shown in Eq. (6). This agreement suggests that our numerical setup outlined above is adequate for the drag calculation intended here. The calculated $\xi_{\|}^{0}$ for the long solid cylinder can also be described by a linear function of zero intercept, $\xi_{\|}^{0}=c 8 \pi \eta x$, where $c$ is a fitting parameter (dashed line). The drag force on a cylinder is found to be slightly larger than that on a spheroid of the same dimension with the correction factor $c \simeq 1.09$.

Motivated by the functional form in Eq. (5), we fit the data shown in Fig. 7 to a function,

$$
\xi_{\|}=\alpha \pi d \eta+\xi_{\|}^{0}+\pi(d / 2) h(2 \eta \rho \omega)^{1 / 2},
$$

where the first term characterizes the contribution from the contact line between the interface and the glass fiber. By dimension, the contact line contribution should be proportional to the total length, $\pi d$, of the contact line and the fluid viscosity $\eta$. The numerical factor $\alpha$ is associated with an integral of the slip velocity profile across the (moving) con-

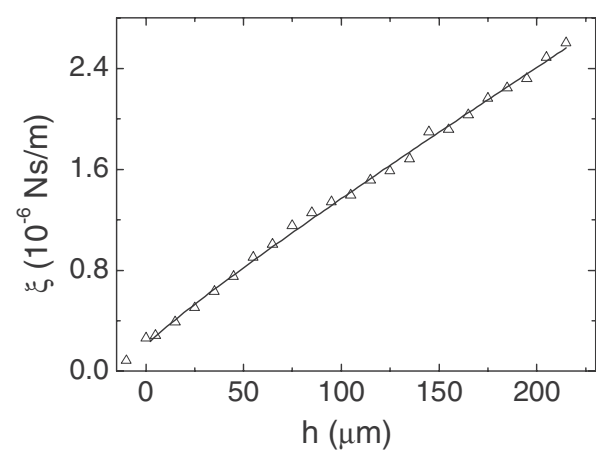

FIG. 9. Fitted values of the friction coefficient $\xi$ as a function of the immersion length $h$. The measurements are made at an interface between air and a 42 wt \% aqueous solution of glycerin. For comparison, the fitted value of $\xi$ in air is also included at $h=-10 \mu \mathrm{m}$. The solid line is a least-square fit of Eq. (9) to the data with $\alpha$ $=6.5 \pm 1$ and $\eta=3.82 \pm 0.08 \mathrm{cP}$.

tact line [34]. The solid line in Fig. 7 is a least-square fit of Eq. (9) to the diamonds using $\alpha$ and $\eta$ as two fitting parameters. In the fitting, we used the numerical result, $\xi_{\|}^{0}$ $=c 8 \pi \eta x$ (see Fig. 8), for the zero-frequency friction coefficient of the long glass fiber. The fitted value of $\eta$ $=1.08 \pm 0.08 \mathrm{cP}$ agrees well with the expect value for water at room temperature. The fitted value of $\alpha=31.2 \pm 4$ is also in the correct range $[34,35]$. The dashed line in Fig. 7 is a plot of Eq. (5), which has a zero intercept and misses the data by a constant offset [i.e., the first term of Eq. (9)]. As mentioned above, the constant offset between the dashed line and the data is caused by the extra drag force imposed by the fluctuating contact line. Figure 7 thus demonstrates that the new hanging-fiber rheometer is indeed capable of measuring a minute amount of the viscous drag produced by a (fluctuating) contact line.

To further test the capability of the hanging-fiber rheometer, we repeat the drag measurements at an interface between air and an aqueous solution of glycerin. The aqueous solution has $42 \mathrm{wt} \%$ mass concentration of glycerin and its viscosity is $3.76 \mathrm{cP}$ at $22.5^{\circ} \mathrm{C}$, which is measured separately by a rheometer (Model ARES, TA instruments/rheometrics). Figure 9 shows the measured friction coefficient $\xi$ as a function of the immersion length $h$. For comparison, we also include the fitted value of $\xi$ in air at $h=-10 \mu \mathrm{m}$. Again, the measured $\xi$ is found to be well described by Eq. (9) and the solid line shows the least-square fit to the data with $\alpha$ and $\eta$ being the two fitting parameters. The fitted value of $\eta$ $=3.82 \pm 0.08 \mathrm{cP}$ agrees well with the independently measured viscosity of the glycerin solution.

The intercept $(\alpha \pi d \eta)$ of the measured $\xi_{\|}$clearly has a nonzero value beyond the experimental uncertainties. We have verified that the experimental uncertainties in determining the contact point $(h=0)$ between the glass fiber and the interface are less than $2 \mu \mathrm{m}$. The linear extrapolation of the measured $\xi_{\|}$to the horizontal $h$ axis, on the other hand, gives rise to a characteristic length $h_{0} \simeq-11 \mu \mathrm{m}$, whose absolute value is much larger than the experimental uncertainties for $h$. In a separate experiment [36], we found that the value of the intercept changes when a small amount of surfactant is added into the liquid, which changes the tension of the three 
interfaces near the contact line. This result further confirms that the contribution to $\alpha \pi d \eta$ comes primarily from the contact line. The experiment thus suggests that the intercept $\alpha \pi d \eta$ is linked to the interfacial hydrodynamics near the contact line.

For high-frequency oscillations, such as that in the present experiment, we find the third term in Eq. (9) becomes dominant over the other two terms. This inertial effect can be described by a modified Reynolds number, $\operatorname{Re}=(d / 2)^{2} \omega / \nu$ $=(1 / 2)(d / \delta)^{2}$, based on the frequency of oscillation $[12,17,31]$. Here $\nu=\eta / \rho$ is the kinematic viscosity of the fluid and $\delta=(2 \nu / \omega)^{1 / 2}$ is the penetration depth of the shear waves. The (modified) Reynolds number measures the relative importance of the time derivative term $\partial_{t} \mathbf{v}$ to the viscous term $\nu \nabla^{2} \mathbf{v}$ in the Stokes equation. For the glass fibers used in the experiment, we have $d \simeq 1.5 \mu \mathrm{m}, \eta \simeq 1 \mathrm{cP}$, and the resonant frequency (in air) $\omega_{0} / 2 \pi \simeq 110 \mathrm{kHz}$. Thus one finds $\delta \simeq 1.7 \mu \mathrm{m}$ and $\mathrm{Re} \simeq 0.4$ for the present experiment. Because the value of $\delta$ is comparable to the fiber diameter $d$, the frequency-dependent inertial contribution to $\xi_{\|}$becomes significant.

\section{CONCLUSION}

We have developed a technique for interfacial microrheology using the AFM as a force sensor. The probe used for microrheology contains a long vertical glass fiber partially immersed through a liquid-air interface. Typical dimensions of the glass fiber used are $1.5 \mu \mathrm{m}$ in diameter and $280 \mu \mathrm{m}$ in length. The top of the glass fiber is glued onto a rectangular shaped cantilever beam, which is mounted on an AFM piezoelectric tube scanner to provide vertical motion with accuracy down to nanometers. The assembly of the modified cantilever is carried out under a high-magnification stereomicroscope using a motorized micromanipulator system. The measured power spectrum $|z(f)|^{2}$ of vertical deflections of the cantilever is sensitive to hydrodynamic interactions between the hanging glass fiber and the liquid-air interface. Such hydrodynamic interactions give rise to the dissipative or viscoelastic response of the fluid to the strain imparted by thermal fluctuations of the modified cantilever.

It is found that the motion of the modified cantilever can be accurately described by the Langevin equation for a damped harmonic oscillator. The experiment verifies the theory and demonstrates its applications. Compared to the large number of AFM applications in the liquid environment, our new technique has several important advantages. (i) Because only a thin glass fiber is in contact with the liquid, the motion of the modified cantilever is not overdamped by the fluid viscosity. As a result, the cantilever's resonant peak has a relatively large $Q$ value. (ii) The use of a thin hanging-fiber as a rheometer probe provides a simple geometry for quan- titative analysis of the rheological property of complex fluids near the interface. (iii) Being operated near its own resonant frequency, the modified cantilever is extremely sensitive to small changes in viscous damping. The technique is capable of measuring a minute amount of viscous stress imposed by the contact line between the interface and the glass fiber. (iv) This technique takes the advantages of a commercial AFM with fast sampling rate up to $5 \mathrm{MHz}$, precise control of the vertical motion of the cantilever down to nanometers, and ease of operation with a friendly computer interface. The experimental uncertainties are essentially statistical. It only takes 2-5 min to collect a power spectrum $|z(f)|^{2}$ with adequate frequency resolution and signal-to-noise ratio. (v) Equation (9) provides a solid base for one to pin point the contact line contribution by simply measuring the jump in drag when the fiber touches the interface. (The drag measurements at different immersion lengths are needed only for the calibration of the hanging-fiber rheometer.) (vi) The hangingfiber probe provides amble flexibility for varying the surface chemistry of the hanging-fiber and the wetting property of the fluids to be studied, making the technique particular useful for the investigation of the contact line dynamics.

As an AFM-based passive microrheometer, the new technique will be useful for the studies of microrheology of liquid-air interfaces and the rheological properties of various complex (bulk) fluids such as colloids, polymers, and emulsions. These studies are of fundamental interest for our general understanding of two- and three-dimensional soft matter systems; they also have immense practical applications in material synthesis, microfluidics and nanotechnology [37]. In addition, the hanging-fiber probe can be readily extended to include a hanging sphere at the end of the glass fiber for the studies of microhydrodynamics or nanohydrodynamics and associated slip phenomena near solid-liquid interfaces, a subject which has attracted much attention in recent years [27,34,38-40]. Furthermore, by using the oscillation control of the AFM cantilever, one can also convert the AFM-based passive microrheometer into an active one. The realization of these experimental advantages will establish a powerful method by which various liquid-liquid interfaces made of polymers, surfactants, and biomolecules (such as membranes) may be characterized at a more quantitative level and with a more precise control of length scales and surface chemistry. The present study is the first step toward this direction.

\section{ACKNOWLEDGMENTS}

We are grateful for useful discussions and communications with M. C. Cross, T.-Z. Qian, X.-P. Wang, D. Ou-Yang, and D. Beck. This work was supported by Research Grants Council of Hong Kong SAR under the Grant No. HKUSTCA05/06.SC01. 
[1] M. Helmer, Nature (London) 437, 206 (2005).

[2] E. Sackmann, Science 271, 43 (1996).

[3] M. Dahan, S. Lévi, C. Luccardini, P. Rostaing, B. Riveau, and A. Triller, Science 302, 442 (2003).

[4] Y. Zhu and S. Granick, Phys. Rev. Lett. 87, 096105 (2001).

[5] Y. Zhu and S. Granick, Phys. Rev. Lett. 88, 106102 (2002).

[6] Jae-Hie J. Cho, B. M. Law, and F. Rieutord, Phys. Rev. Lett. 92, 166102 (2004).

[7] D. E. Aston and J. C. Berg, J. Colloid Interface Sci. 235, 162 (2001).

[8] G. Y. Chen, R. J. Warmack, T. Thundat, D. P. Allison, and A. Huang, Rev. Sci. Instrum. 65, 2532 (1994).

[9] J. K. Horber and M. J. Miles, Science 302, 1002 (2003).

[10] A. Roters, M. Gelbert, M. Schimmel, J. Rühe, and D. Johannsmann, Phys. Rev. E 56, 3256 (1997).

[11] A. Roters, M. Schimmel, J. Rühe, and D. Johannsmann, Langmuir 14, 3999 (1998).

[12] R. Rajagopalan, Colloids Surf., A 174, 253 (2000).

[13] J. E. Sader, J. Appl. Phys. 84, 64 (1998).

[14] R. J. Clarke, O. E. Jensen, J. Billingham, A. P. Pearson, and P. M. Williams, Phys. Rev. Lett. 96, 050801 (2006).

[15] M. R. Paul and M. C. Cross, Phys. Rev. Lett. 92, 235501 (2004).

[16] M. R. Paul, M. T. Clark, and M. C. Cross, Nanotechnology 17, 4502 (2006).

[17] H.-L. Ma, J. Jimenez, and R. Rajagopalan, Langmuir 16, 2254 (2000).

[18] A. Mehta, S. Cherian, D. Hedden, and T. Thundata, Appl. Phys. Lett. 78, 1637 (2001).

[19] P. I. Oden, G. Y. Chen, R. A. Steele, R. J. Warmack, and T. Thundat, Appl. Phys. Lett. 68, 3814 (1996).

[20] F. Reif, Fundamentals of Statistical and Thermal Physics (McGraw-Hill, Auckland, 1985).

[21] K. Berg-Sørensen and H. Flyvbjerg, Rev. Sci. Instrum. 75, 594 (2004).

[22] D. A. Walters, J. P. Cleveland, N. H. Thomson, P. K. Hansma, M. A. Wendman, G. Gurley, and V. Elings, Rev. Sci. Instrum. 67, 3583 (1996).
[23] W. Chen, S.-S. Tan, Z.-S. Huang, T.-K. Ng, W. T. Ford, and P. Tong, Phys. Rev. E 74, 021406 (2006).

[24] P. Attard and S. J. Miklavcic, J. Colloid Interface Sci. 247, 255 (2002).

[25] The Thermal PSD software provided by Asylum Research Inc. uses the normalization, $\left\langle z^{2}(t)\right\rangle_{t}=\int_{0}^{\infty}|z(f)|^{2} d f$, for its measured $|z(f)|^{2}$. The factor of 2 difference in the definition of $|z(f)|^{2}$ has been corrected in the presentation of data shown in this paper.

[26] A. Prevost, E. Rolley, and C. Guthmann, Phys. Rev. Lett. 83, 348 (1999).

[27] P.-G. de Gennes, F. Brochard-Wyart, and D. Quéré, Capillarity and Wetting Phenomena (Springer, New York, USA, 2004).

[28] S. Ramos and A. Tanguy, Eur. Phys. J. E 19, 433 (2006).

[29] L. G. Leal, Advanced Transport Phenomena (Cambridge University Press, Cambridge, UK, 2007), p. 557.

[30] M. Doi and S. F. Edwards, The Theory of Polymer Dynamics (Oxford University Press, Oxford, UK, 1986).

[31] L. D. Landau and E. M. Lifshitz, Fluid Mechanics, 2nd ed. (Butterworth-Heinemann, Oxford, UK, 1987).

[32] R. J. Clarke, O. E. Jensen, J. Billingham, and P. M. Williams, Proc. R. Soc. London, Ser. A 462, 913 (2006).

[33] In Ref. [17], the inertial correction to the friction coefficient $\xi_{\perp}$ perpendicular to the axis of a long cylinder is considered. It can be shown that the inertial correction to $\xi_{\|}$is a factor of 2 smaller than that to $\xi_{\perp}$.

[34] T.-Z. Qian, X.-P. Wang, and P. Sheng, Phys. Rev. Lett. 93, 094501 (2004).

[35] T.-Z. Qian, X.-P. Wang, and P. Sheng, Phys. Rev. E 72, 022501 (2005).

[36] Xiong et al. (unpublished).

[37] The World of Nano-Biomechanics, edited by A. Ikai (Elsevier, Amsterdam, The Netherlands, 2008).

[38] J. Eggers, Phys. Fluids 17, 082106 (2005).

[39] C. C. Cottin-Bizonne, B. Cross, A. Steinberger, and E. Charlaix, Phys. Rev. Lett. 94, 056102 (2005).

[40] T.-Z. Qian, X.-P. Wang, and P. Sheng, J. Fluid Mech. 564, 333 (2006). 